\title{
AN ULTRASTRUCTURAL STUDY OF THE REVERSIBILITY OF THE EFFECTS OF HYPOXIA ON HUMAN TROPHOBLAST MAINTAINED IN ORGAN CULTURE
}

\author{
ALASTAIR H. MACLENNAN, * MATTHEW J. GARTY, \\ BRIAN L. SHEPPARD* AND FRANK SHARP \\ *Nuffeld Department of Obstetrics and Gynaecology, Fohn Radcliffe Hospital, Oxford, \\ and University Department of Obstetrics, Queen Mother's Hospital, Glasgow
}

(Received 7th November 1974)

\begin{abstract}
Summary. Ultrastructural changes in normal human trophoblast maintained in hypoxic organ culture are described. Marked structural changes were noted even after hypoxia for $6 \mathrm{hr}$. These changes could be partly reversed by reoxygenation after hypoxia for $18 \mathrm{hr}$ or less, but no regeneration occurred when hypoxia lasted for $24 \mathrm{hr}$ or longer. The significance of the vulnerability of trophoblast to hypoxia is discussed.
\end{abstract}

\section{INTRODUCTION}

Placental villi can be maintained in organ culture for over 10 days without morphological changes that can be detected by light microscopy (Gerbie, Hathaway \& Brewer, 1966; Hou, Ewen \& Beck, 1968; Fox, 1970), and for at at least 4 days without significant ultrastructural changes (MacLennan, Sharp \& Shaw Dunn, 1972). When trophoblast is cultured in hypoxic conditions, marked changes occur in its structure (Fox, 1970; MacLennan et al., 1972), and histochemical studies have shown that cellular metabolism is significantly curtailed (Sharp, Carty \& Young, 1972). Ultrastructural comparison of trophoblast grown in hypoxic culture showed that the changes induced by these conditions were very similar to those occurring in placental villi from patients with pre-eclampsia and from patients with unexplained placental insufficiency (MacLennan et al., 1972). It was suggested that such placental changes in these diseases may be due to hypoxia and that oxygen-dependent enzyme systems in the trophoblast were inactivated, either temporarily or permanently.

The purpose of this investigation was to see if the structural changes induced by hypoxia in trophoblast are reversible and, if so, for how long the tissue could withstand such a deprivation.

\section{MATERIALS AND METHODS}

Placentae from patients with uncomplicated pregnancies at 38 to 41 weeks of gestation were collected and processed within $2 \mathrm{~min}$ of vaginal delivery. Under aseptic conditions, portions of placental villous tissue not greater than $2 \mathrm{~mm}$ in 
diameter were dissected free. The tissue fragments were washed in sterile physiological saline three times and then transferred to the incubation system, except for a few villi that were fixed immediately for examination to ensure that they were ultrastructurally normal and suitable for use as controls for the villi being cultured (Pl. 1, Fig. 1).

\section{Incubation system}

The technique of culture was a modification of that described by Fox \& Kharkongor (1970), as previously described (MacLennan et al., 1972). Tissue from the same placenta was placed in twelve culture jars and each jar was initially blown through for $15 \mathrm{~min}$ with a gaseous mixture containing $6 \%$ oxygen, $5 \%$ carbon dioxide and $89 \%$ nitrogen. The gas ports were then closed and the jars, with this gaseous mixture, were transferred to an incubator at $37^{\circ} \mathrm{C}$. Pairs of jars were removed at $6,12,18,24,48$ and $72 \mathrm{hr}$.

After removal of each pair of cultures at the appropriate time, tissues from one culture were fixed for electron microscopy whilst the other jar was blown through for 15 min with a gaseous mixture containing $95 \%$ oxygen and $5 \%$ carbon dioxide. The jar was then incubated for a further $24 \mathrm{hr}$ at $37^{\circ} \mathrm{C}$ before being opened, when the 'reoxygenated' tissue was removed and fixed for electron microscopy.

\section{Electron microscopy}

The specimens were fixed by immersion in $5 \%$ glutaraldehyde in phosphate buffer ( $\mathrm{pH} 7 \cdot 4,4^{\circ} \mathrm{C}$ ) for $1 \mathrm{hr}$ and washed in phosphate buffer for $48 \mathrm{hr}$. The tissues were then post-fixed in phosphate-buffered $1 \%$ osmium tetroxide for 1 $\mathrm{hr}$, dehydrated in a graded series of ethanol and embedded in Araldite. After slow polymerization, $1 \mu \mathrm{m}$ sections were prepared and stained with methylene blue and examined under a light microscope. Representative thin sections cut on an L.K.B. Ultrotome were stained with uranyl acetate and lead citrate (Reynolds, 1963) and examined with an A.E.I. EM6B electron microscope. The changes in the trophoblast were similar to those which have already been described after hypoxia for 24 to $96 \mathrm{hr}$ (MacLennan et al., 1972) except that the changes at $6 \mathrm{hr}$ were less pronounced and were thereafter progressive with the duration of hypoxia.

\section{RESULTS}

\section{Trophoblast cultured in $6 \%$ oxygen}

Degeneration of the trophoblast could be detected even in the specimens which had been exposed to the shortest period of hypoxia, $6 \mathrm{hr}$.

The ultrastructural changes included thickening of the basal lamina, progressive vacuolation and thinning of the syncytiotrophoblast, multiplication of the syncytial nuclei with marked clumping of the nuclear chromatin at the periphery of the nucleus with apparent loss of some of the chromatin, enlargement of the subtrophoblastic space, a gradual degeneration of the mitochondria, Golgi complexes and endoplasmic reticulum, an increase in lysosome-like bodies and, lastly, a change in the maternal surface profile with cytoplasmic projections and 
$\mathrm{PI} \triangle \mathrm{ATE}, 1$

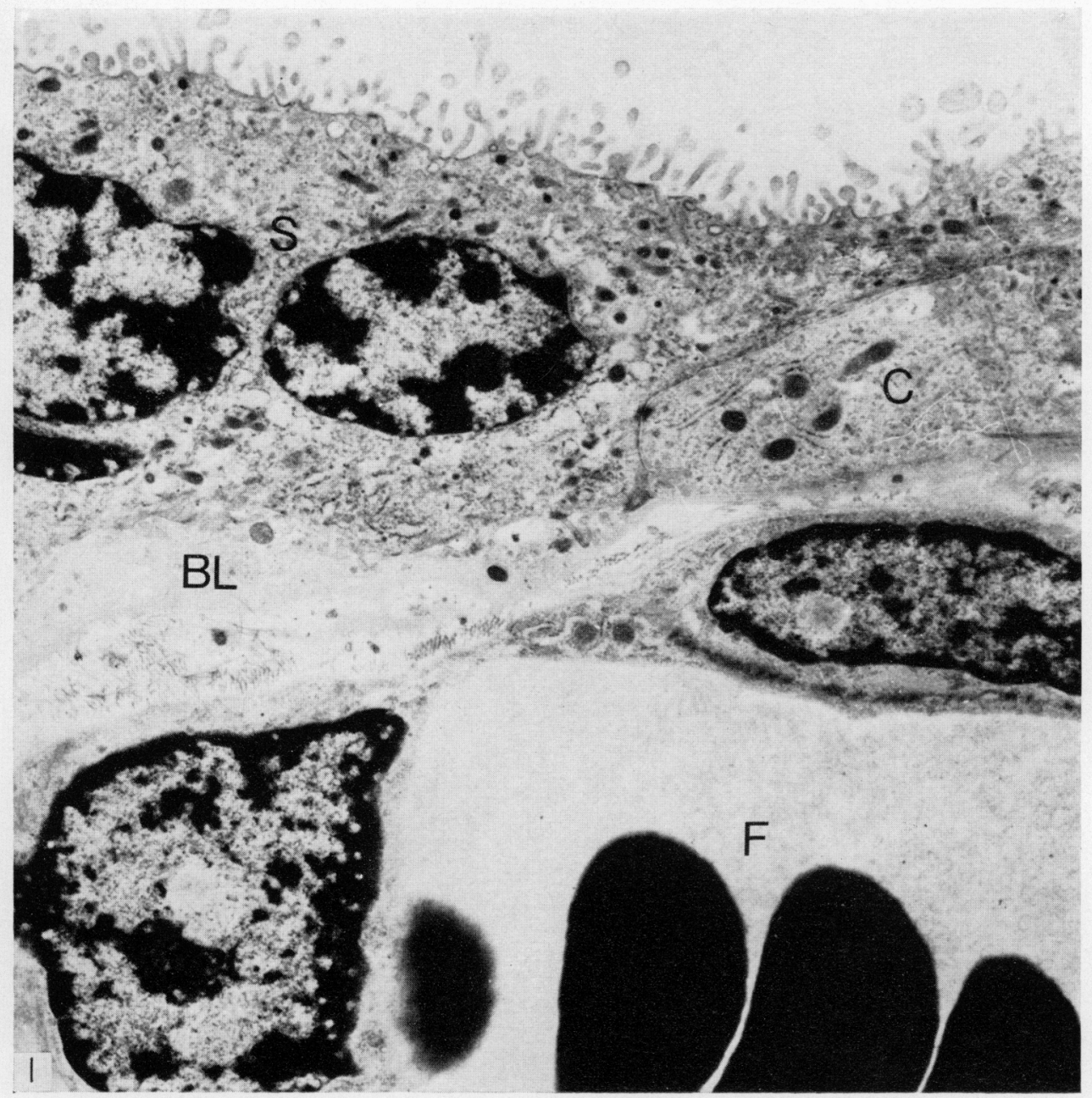

Fig. I. Normal placental villus at term before transfer to organ culture showing syncytiotrophoblast (S), cytotrophoblast (G), basal lamina (BL) and a fetal capillary (F). $\times 11,500$. 


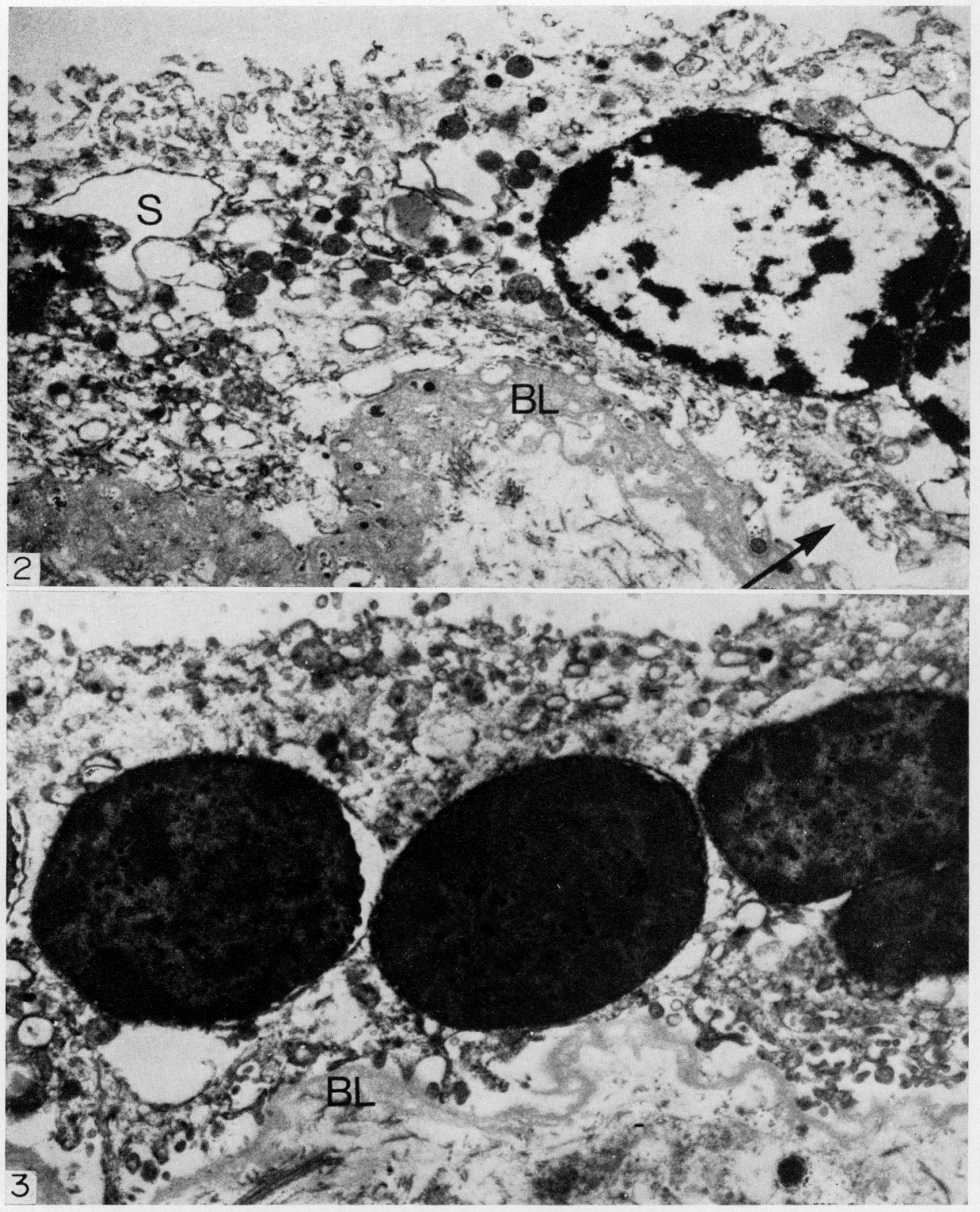

Fir. 2. Trophoblast after organ culture in $6 \%$ oxygen for $18 \mathrm{hr}$. In this hypoxic environment the syncytium (S) shows increased vacuolation and loss of eytoplasmic organclles. The basal lamina (BI) is thickened and the subtrophoblastic space (arrow) is enlarged. The nuclear chromatin has become more sparse and clumped at the periphery of the nuclei. $\times 11,500$.

Fic. 3. Trophoblast after organ culture in $6 \%$ oxygen for $18 \mathrm{hr}$ (sce Fig. 2) and subsequent reoxygenation in $95 \%$ oxygen for $24 \mathrm{hr}$. Multiplication of the syncytial nuclei has occurred. associated with greater dispersion of the chromatin. The basal lamina (BL) has thinned and there is less cyloplasmic vacuolation. $\times 11,500$. 
PI. \T'L: 3

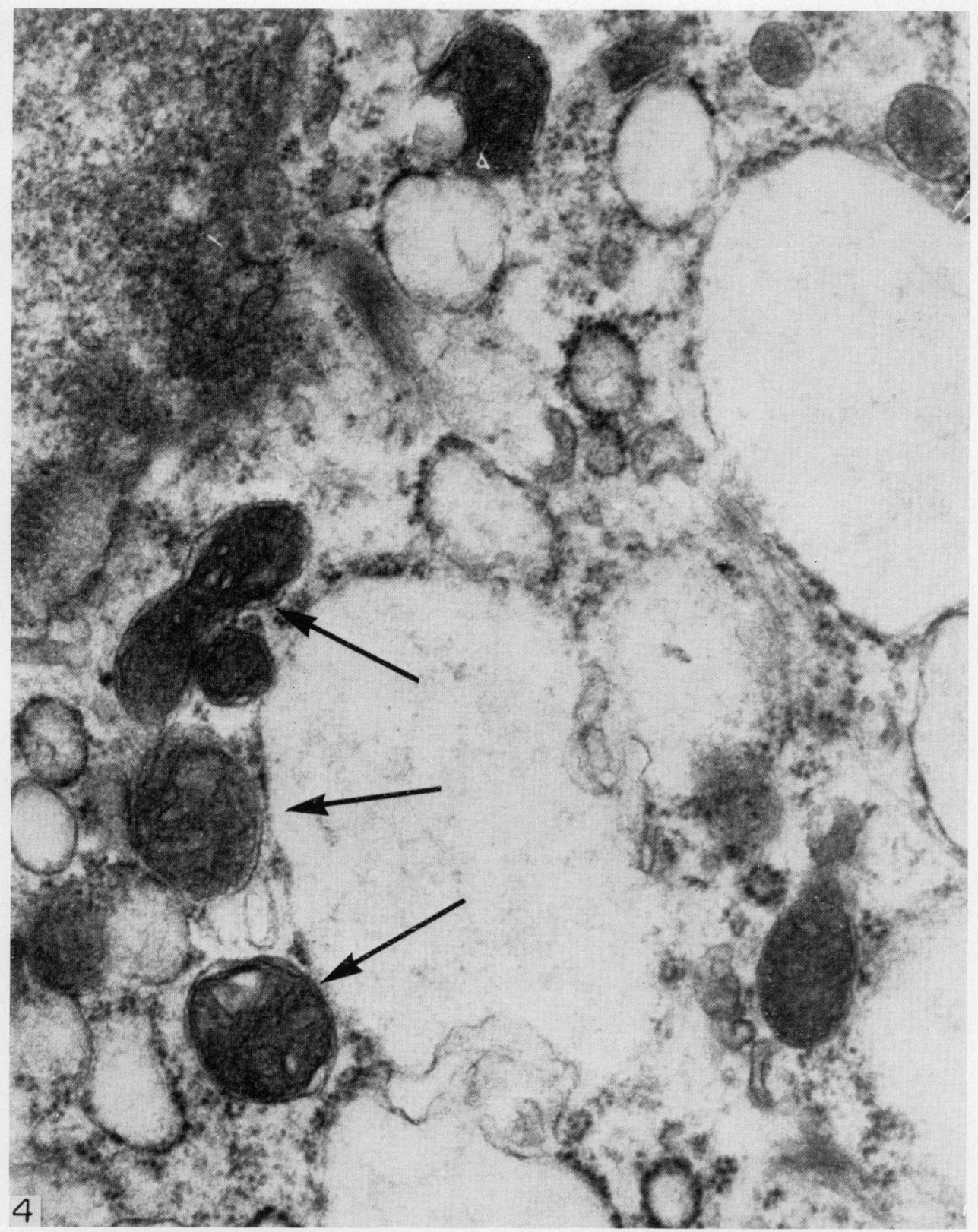

Fic. 4. High-power electron micrograph of cytoplasm of trophoblast reoxygenated after $18 \mathrm{hr}$ of hypoxia. The mitochondria (arrows) that before reoxygenation were hardiy recognizable have now regained some of their normal structure. $\times 45,000$. 


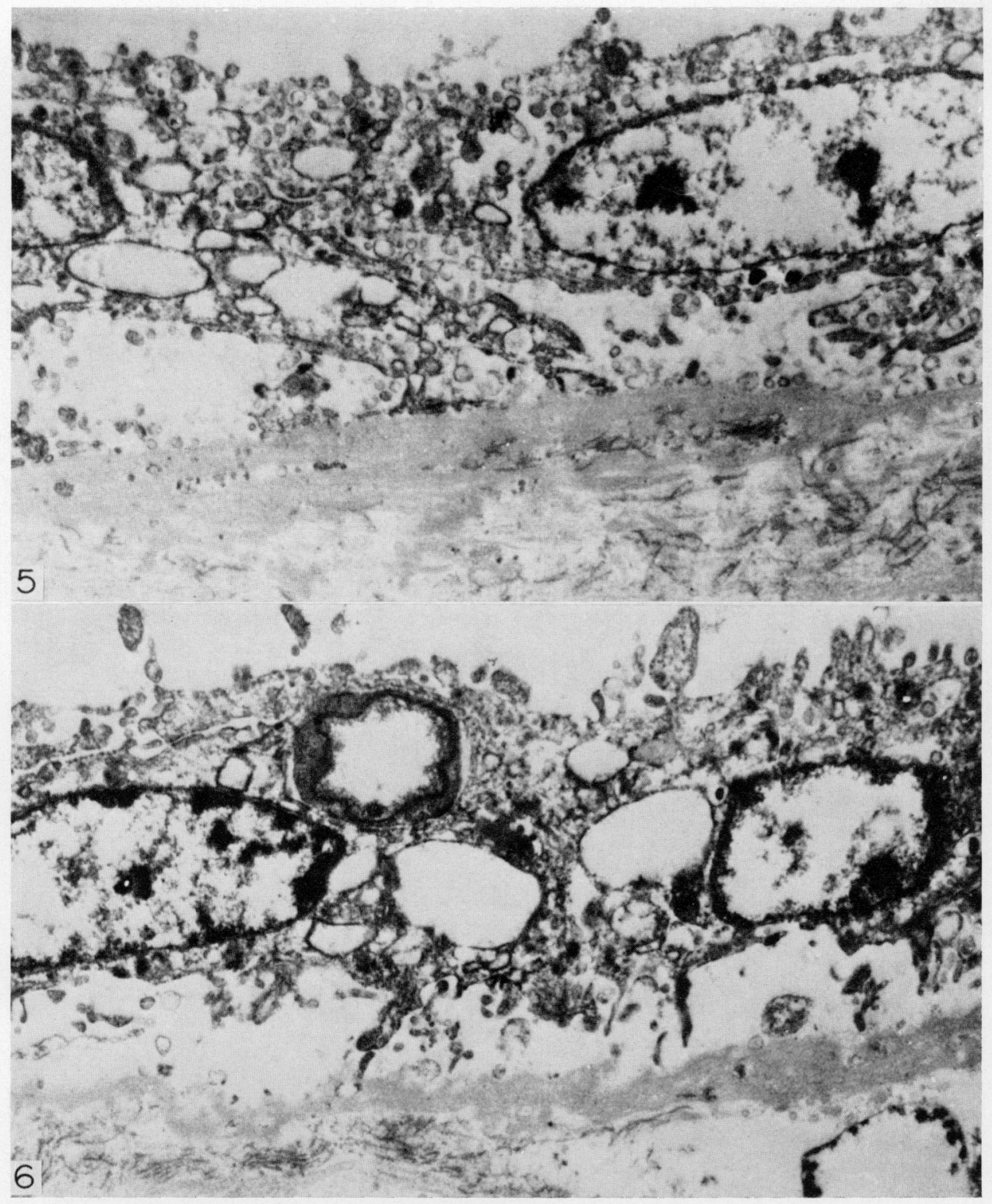

Fic. 5. Trophoblast after organ culture in $6 \%$ oxygen for 48 hr. Marked degeneration of the syncytium has occurred. $\times 11,500$.

Fra. 6. Trophoblast after organ culture in $6 \%$ oxygen for $48 \mathrm{hr}$ (see Fig. 5) and subsequent rexygenation for $24 \mathrm{hr}$. No significant changes have occurred. $\times 11,500$. 
clubbing of the microvilli (PI. 2, Fig. 2 and Pl. 4, Fig. 5). With increasing hypoxia, the trophoblast became more degenerate and signs of normal intracellular activity were lacking (Pl. 4, Fig. 5).

\section{Reoxygenated trophoblast after hypoxic culture}

Consistent changes were noted in all specimens reoxygenated after hypoxia for 6, 12 and $18 \mathrm{hr}$ (Pl. 2, Fig. 3). Changes were not seen in any of the specimens that had been subjected to hypoxia for $24 \mathrm{hr}$ or more (Pl. 4, Figs 5 and 6 ). The structural reorganization was similar in the 6-, 12- and 18-hr specimens.

The most notable change was a consistent multiplication of the syncytial nuclei with frequent syncytial knot formation. Nuclear counts during scanning confirmed this impression. No further loss of nuclear chromatin occurred and it appeared more evenly dispersed throughout the nuclei, there being more euchromatin and less heterochromatin than before reoxygenation (Pl. 2, Fig. 3). The basal lamina was generally about four times thinner than in the corresponding hypoxic specimens. The structure of the cell organelles seemed clearer and cristae in the mitochondria were visible (Pl. 3, Fig. 4). The mitochondria and endoplasmic reticulum were more numerous in the reoxygenated specimens, but there was no change in the number of lysosome-like bodies. The syncytiotrophoblast was less vacuolated and thus the cell organelles were more dense.

Comparison of the tissues reoxygenated after hypoxia for 24,48 or $72 \mathrm{hr}$ with their hypoxic counterparts showed no significant changes in any of the specimens examined (Pl. 4, Figs 5 and 6).

\section{DISCUSSION}

This study confirms the findings of the previous investigation (MacLennan et al., 1972) of the ultrastructural changes induced by hypoxia in the trophoblast. In the present study, however, the effects of shorter periods of hypoxia were investigated; even the shortest period of hypoxia caused major intracellular changes. Hypoxic episodes of $6 \mathrm{hr}$ or even less may, therefore, significantly damage trophoblast, a possibility according with the findings of Sharp et al. (1972) who found that hydroxysteroid dehydrogenase (HSD) activity in trophoblast was markedly diminished even after $6 \mathrm{hr}$ in hypoxic culture.

Some regeneration of trophoblast occurred with reoxygenation after as much as $18 \mathrm{hr}$ hypoxia, but no evidence of reversibility was seen in tissue that had been subjected to hypoxia for $24 \mathrm{hr}$ or more. Sharp et al. (1973) found that $17 \beta$-HSD activity could be re-activated by reoxygenation after hypoxia of up to $12 \mathrm{hr}$ but not after hypoxia for $24 \mathrm{hr}$. The inhibition of enzyme activity in hypoxic trophoblast would appear to be related to the degeneration of the mitochondria and loss of endoplasmic reticulum in these conditions. Changes in oxygen tension or hydrogen ion concentration presumably inhibit oxidative phosphorylation within the mitochondria, and the ensuing lack of energy for normal trophoblast functions, such as transcellular transport and hormone production, causes an accumulation of absorbed material within the syncytiotrophoblast. This probably accounts for the increasing vacuolation of the syncytiotrophoblast during hypoxia. Such an hypothesis is supported by the fact that the 
vacuolation in villi subjected to shorter periods of hypoxic culture appeared to be reduced after reoxygenation and was associated with reappearance of the cristae in the mitochondria and an increase in endoplasmic reticulum. Hypoxia for $24 \mathrm{hr}$ or more appeared to cause irreversible damage to the trophoblast enzyme systems because the amount of vacuolation in these samples was not affected by reoxygenation.

There was a marked difference in the appearance of the cell nuclei in the reoxygenated specimens previously subjected to $18 \mathrm{hr}$ of hypoxia, but it was not possible to determine whether this change represented signs of regeneration. In general, it is believed that nuclei containing large clumps of densely staining heterochromatin are metabolically less active than those containing evenly distributed euchromatin which stains more lightly (Fawcett, 1966).

The changes in healthy trophoblast subjected to hypoxia in vitro have been shown to be very similar to the placental changes of pre-eclampsia and the placentae of babies that are 'small-for-their-dates' (MacLennan et al., 1972). The finding of a possible reversibility of these early hypoxic changes in vitro but their irreversibility soon after $18 \mathrm{hr}$ suggests that it would be difficult to improve placental transfer once these clinical syndromes were established.

\section{ACKNOWLEDGMENTS}

We are grateful for the expert technical help of Mrs Uta Sheppard in the preparation of the tissue specimens for electron microscopy and also thank Professor Alec C. Turnbull and Mr John Bonnar for their advice and encouragement in the preparation of this paper.

\section{REFERENCES}

Fawcetr, D. W. (1966) The Cell, p. 8. Saunders, Philadelphia.

Fox, H. (1970) Effect of hypoxia on trophoblast in organ culture. Am. F. Obstet. Gynec. 107, 1058-1064.

Fox, H. \& KHARKONGOR, N. F. (1970) The effect of hypoxia on the enzyme histochemistry of placental villi maintained in organ culture. F. Obstet. Gynaec. Br. Commonw. 77, 526-530.

Gerbie, A. B., Hathaway, H. R. \& BreWer, J. I. (1966) Organ culture of trophoblast. Obstet. Gynec., $\mathcal{N} . X .31,151-156$.

Hov, L. T., EWEN, S. W. B. \& BEck, J. S. (1968) Histological, metabolic and histochemical studies on normal human placenta in organ culture. Br. F. exp. Path. 49, 648-657.

MacLinnan, A. H., Sharp, F. \& Shaw Dunn, J. (1972) The ultrastructure of human trophoblast in spontaneous induced hypoxia using a system of organ culture. F. Obstet. Gynaec. Br. Commonw. 79, 113-121.

REynolds, E. S. (1963) The use of lead citrate at high $\mathrm{pH}$ as an electron-opaque stain in electron microscopy. F. Cell Biol. 17, 208-212.

Sharp, F., GarTy, M. J. \& Young, H. (1972) The effects of hypoxia on hydroxysteroid dehydrogenase activity in placental villi maintained in organ culture. F. Obstet. Gynaec. Br. Commonw. 79, 44-49.

Sharp, F., Garty, M. J. \& Young, H. (1973) The reversibility of the effects of hypoxia on hydroxysteroid dehydrogenase (HSD) activity in placental villi maintained in organ culture. $\mathcal{F}$. Obstet. Gynaec, Br. Commonw. 80, 325-328. 